\title{
Clinical Characteristics of Aortic Aneurysm in MIMIC-III
}

\author{
Kun Song, ${ }^{*}$ Cuirong Guo, ${ }^{*}$ Kongzhi Yang, ${ }^{*}$ Changluo Li, ${ }^{\dagger}$ Ning Ding ${ }^{\dagger}$ \\ Department of Emergency Medicine, Changsha Central Hospital, University of South China, Hengyand, Hunan, China
}

\section{ABSTRACT}

Background: Aortic aneurysm (AA) is an aortic disorder prone to sudden, life-threatening aortic dissection or rupture, with poor clinical outcomes. In this study, we aimed to analyze the clinical characteristics of AA in MIMIC-III to explore implications for management.

Methods: All patients with AA, including abdominal aortic aneurysm (AAA) and thoracic aortic aneurysm (TAA), in the MIMIC-III database were included. Clinical and laboratory variables were analyzed and compared in AAA and TAA.

Results: A total of 345 patients, including 183 patients with AAA and 162 patients with TAA, were enrolled in this study. The in-hospital mortality in AAA and TAA groups was $6.01 \%$ and $3.7 \%$, respectively. In the nonsurvivor groups in both AAA and TAA, patients were older, and the incidence of surgery was lower. In the nonsurvivor group of AAA, the levels of alanine aminotransferase, aspartate aminotransferase, urea nitrogen, creatinine, lactate dehydrogenase (LDH), creatine kinase, anion gap, and lactate were significantly higher in the nonsurvivor group, whereas the level of albumin was lower. In the nonsurvivor group of TAA, the level of LDH significantly increased and the level of albumin decreased. In the nonsurgery group, in-hospital mortality was higher, and patients were older, with higher levels of glucose, total bilirubin, urea nitrogen, and creatinine and longer length of stay in ICU and hospital.

Conclusion: Age, surgery, albumin, and LDH showed significant differences between survivor and nonsurvivor groups in both AAA and TAA. In the nonsurgery group, the mean age was older and disease severity was worse, with poorer clinical outcomes. Older AA patients without surgery and with lower levels of albumin and higher levels of $\mathrm{LDH}$ had higher risk of in-hospital mortality.

Received December 20, 2020; received in revised form February 17, 2021; accepted February 18, 2021.

Correspondence: Changluo Li or Ning Ding, Department of Emergency Medicine, Changsha Central Hospital, University of South China, No. 161 Shaoshan South Road, Changsha, Hunan, 410004, China; 86 731-85667935 (e-mail: 813205232@qq.com [C. Li] or doctordingning@csu.edu.cn, doctordingning@sina.com [N. Ding]).

*K. Song, C. Guo, and K. Yang contributed equally to this article.

†C. Li and N. Ding are co-authors for correspondence.

\section{INTRODUCTION}

Aortic aneurysm (AA) is an aortic disorder prone to sudden, life-threatening aortic dissection or rupture, with poor clinical outcomes [Isselbacher 2005]. Abdominal aortic aneurysm (AAA) and thoracic aortic aneurysm (TAA) are subgroups based on anatomic characteristics. Etiology [Li 2018; Mori 2020], pathogenesis of aneurysm formation [Yamada 2020; Pasta 2020], clinical manifestation, management, and prognosis [Yigit 2020; Wang 2020] differ between AAA and TAA.

In an increasing number of retrospective and observational studies, different scoring systems [Pires Coelho 2019], plasma biomarkers [Molecek 2019; Acosta 2018], and machine learning methods [Jordanski 2018] have been applied to evaluate the clinical outcomes of AA in different samples. Previous studies mainly focused on AA patients after different surgeries and discussed the association of surgeries with clinical outcomes [Grootes 2018; Leighton 2019]. In this study, AA in the MIMIC-III public database was retrospectively analyzed to explore differences in clinical characteristics in AAA and TAA. We aimed to identify those patients with higher risk of poor outcomes. Risk factors for in-hospital mortality were further evaluated to provide useful clinical guidance for physicians so that early prevention and management may be implemented in AA patients with higher risk for fatal outcomes in hospital.

\section{METHODS}

\section{Ethics Approval and Consent to Participate}

MIMIC-III is an anonymized public database. To apply for access to the database, we passed the Protecting Human Research Participants examination (no. 32900964). The project was approved by the institutional review boards of MIT and Beth Israel Deaconess Medical Center and was given a waiver of informed consent.

\section{Dataset}

All patients with AA including TAA and AAA (International Classification of Diseases, Revision 9 codes 4411, 4412, 4413, 4414) in the MIMIC-III database were included in this study. MIMIC-III is a large US-based critical care public database containing data associated with 53,423 adult patients $(\geq 16$ years old) from 2001 to 2012 and 7870 neonates from 2001 to 2008 in intensive care units (ICUs) [Johnson 2016]. Data include vital signs, medications, laboratory measurements, observations, and provider notes charted by care providers, and fluid balance, procedure codes, diagnostic codes, imaging 
reports, hospital lengths of stay, and survival data are comprehensively recorded. The following tables from MIMIC III dataset were applied in our study: ADMISSIONS, CHARTEVENTS, D_ICD DIAGNOSIS, D_ITEMS, D_ LABIEVENTS, DIAGNOSIS_ICD, ICUSTAYS, LABEVENTS, NOTEEVENTS, PATIENTS, INPUTEVENTS_ CV, INPUTEVENTS_MV, and OUTPUTEVENTS.

\section{Data Extraction}

Structured Query Language (SQL) with PostgreSQL tools was implemented for data extraction. General characteristics, vital signs, and laboratory variables of AA from MIMIC-III were extracted for further analysis. General characteristics including age, sex, marital status, ethnicity, and admission type were extracted. Vital signs including systolic blood pressure (SBP), diastolic blood pressure (DBP), and heart rate (HR) were extracted within 24 hours after admission. The following comorbidities were extracted: diabetes, hypertension, renal disease, and coronary heart disease (CHD). The following laboratory variables within 24 hours after admission were included in this study: white blood cells (WBC), platelets (PLT), mean corpuscular volume (MCV), red cell volume distribution width (RDW), hematocrit (HCT), glucose, prothrombin time (PT), thrombin time (TT), albumin, alanine aminotransferase (ALT), aspartate aminotransferase (AST), total bilirubin, urea nitrogen, creatinine, lactate dehydrogenase (LDH), creatine kinase (CK), total calcium, sodium, anion gap, lactate, and triglycerides. When a variable was recorded outside the initial 24 hours, the first instance was recorded in the study. Clinical outcomes, including length of stay (LOS) in ICU and in-hospital mortality, and scoring systems, including Sequential Organ Failure Assessment (SOFA) and Acute Physiology and Chronic Health Evaluation (APACHEII), were also extracted.

\section{Statistical Analysis}

All variables are presented as mean (standard deviation [SD]) or median (interquartile range [IQR]) for continuous variables, and categorical variables are presented as frequencies and percentages. Comparison between 2 groups was performed by $\chi^{2}$ test or Mann-Whitney $U$ test.

\section{Availability of Data and Materials}

Datasets used in the present study are available from the corresponding author on reasonable request.

\section{RESULTS}

\section{General Characteristics of Patients with Aortic Aneurysm}

A total of 345 patients were enrolled in this study, including 183 patients with AAA and 162 with TAA (Figure 1). General characteristics of AAA and TAA groups are shown in Table 1. In-hospital mortality for AAA and TAA was $6.01 \%$ and $3.7 \%$, respectively. Median age was 73 years in AAA and 67 in TAA. There was a significant difference in the proportion of males between the groups $(68.85 \%$ versus $54.93 \%, P$ $=.008)$. In both groups, $>50 \%$ of patients were married, and most patients were American. In admission type, elective was

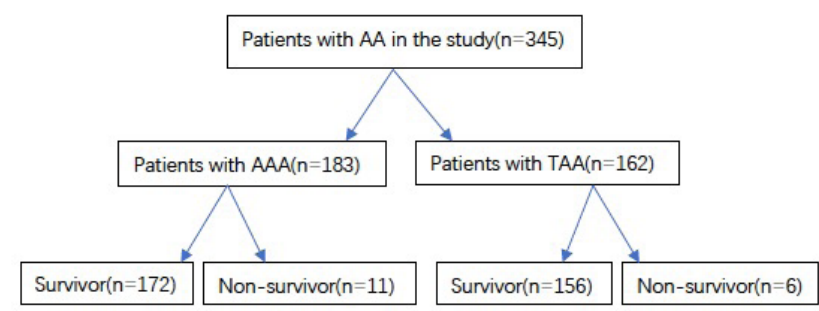

Figure 1 :Flow chart for patients enrollment and study design. Abbreviations: $\mathrm{AA}=$ aortic aneurysm, $\mathrm{AAA}=\mathrm{abdominal}$ aortic aneurysm, $\mathrm{TAA}=$ thoracic aortic aneurysm

Figure 1. Flow chart of patient enrollment and study design.

the most common, accounting for $72.13 \%$ and $69.75 \%$ of AAA and TAA, respectively. However, $30 \%$ of AA patients were admitted to the hospital emergently or urgently. In the AAA group, LOS in ICU was significantly longer than that in the TAA group $(P<.001)$. Scores of APACHEII $(P=.035)$ and SOFA $(P=.045)$ were higher for AAA.

\section{Comparison of Variables between Survivor and Nonsurvi- vor Groups in $A A A$}

A comparison of variables in AAA is shown in Table 2. In the nonsurvivor group, patients were older $(P=.045)$. There was no significant difference in proportion of males, SBP, DBP, or HR between the groups. In the survivor group, the numbers of patients with diabetes, hypertension, renal disease, and CHD were $4(2.32 \%), 99(57.55 \%), 4(2.32 \%)$, and $42(24.21 \%)$, respectively. In the nonsurvivor group, the numbers of patients with hypertension and renal disease were 2 (18.18\%) and 1 $(9.09 \%)$, respectively. No patients had diabetes or coronary heart disease in the nonsurvivor group. The incidence of surgery in the survivor group was $5.81 \%$, and none of the patients in the nonsurvivor group had surgery $(P<.001)$.

There was no significant difference in WBC $(P=.313)$, PLT $(P=.942), \operatorname{MCV}(P=.913), \operatorname{RDW}(P=.799)$, HCT $(P=.165)$, glucose $(P=.429), \mathrm{PT}(P=.633)$, TT $(P=.574)$, total bilirubin $(P=.139)$, total calcium $(P=.273)$, sodium $(P$ $=.359)$, or triglycerides $(P=.787)$ between the groups. The levels of $\operatorname{ALT}(P=.006)$, AST $(P=.033)$, urea nitrogen $(P=$ $.016)$, creatinine $(P=.018), \mathrm{LDH}(P=.001), \mathrm{CK}(P=.024)$, anion gap $(P=.017)$, and lactate $(P=.045)$ were significantly higher in the nonsurvivor group, whereas the level of albumin was lower $(P=.016)$. LOS in ICU was longer in the nonsurvivor group $(P=.022)$. The scores of APACHEII $(P=.167)$ and SOFA $(P=.304)$ showed no difference between the groups.

\section{Comparison of Variables between Survivor and Nonsurvi- vor Groups in TAA}

A comparison of variables in TAA is shown in Table 3. Patients in the nonsurvivor group were older $(P=.04)$. The proportion of males, SBP, DBP, and HR showed no significant difference. There was also no difference in diabetes, hypertension, or CHD. Surgery was implemented in $89.1 \%$ of patients in the survivor group and only 4 in the nonsurvivor group $(P=.014)$. 
In the nonsurvivor group, the level of $\mathrm{LDH}$ was significantly increased $(P<.001)$, and the level of albumin was decreased $(P=.005)$. Other laboratory variables including $\operatorname{WBC}(P=.248), \operatorname{PLT}(P=.301), \operatorname{MCV}(P=.328), \operatorname{RDW}(P=$ $.596)$, HCT $(P=.361)$, glucose $(P=.949)$, PT $(P=.893)$, TT $(P=.356)$, ALT $(P=.356)$, AST $(P=.867)$, total bilirubin $(P$ $=.085)$, urea nitrogen $(P=.355), \mathrm{CK}(P=.153)$, total calcium $(P=.576)$, sodium $(P=.266)$, anion gap $(P=.279)$, lactate $(P$ $=.351)$, and triglycerides $(P=.109)$ showed no significant difference between the groups.

\section{Comparison of Variables between Surgery and Nonsurgery Groups Overall}

Between surgery and nonsurgery groups, variables are compared in Table 4 . In the surgery group, patients were younger, and the proportion of males was lower compared with the nonsurgery group (both $P<.001$ ). More than $90 \%$ of patients with TAA had surgery, versus $<10 \%$ with AAA $(P<.001)$. Insignificant differences were found in vital signs including $\operatorname{SBP}(P=.099)$, DBP $(P=.761)$, and HR $(P=.212)$.
Significant differences were shown in glucose $(P<.001)$, albumin $(P<.001)$, total bilirubin $(P=.015)$, urea nitrogen $(P<$ $.001)$, and creatinine $(P<.001)$. In the surgery group, LOS in ICU was shorter $(P=.015)$ and in-hospital mortality was significantly lower. Both APACHEII $(P<.001)$ and SOFA $(P$ $=.024)$ scores were significantly lower in the surgery group.

\section{DISCUSSION}

In our study, age, surgery, albumin, and LDH showed significant differences between survivors and nonsurvivors in both AAA and TAA. In the nonsurvivor group, the mean age was older, the proportion of patients with surgery was lower, the level of albumin was decreased, and the level of LDH was elevated. In the nonsurgery group, the mean age was older, and disease severity was worse, with poorer clinical outcomes.

Age has been identified as a risk factor in many disorders as in AA [Fattahi 2020]. Those older than 59 years with stroke or a transient ischemic attack were found to have a doubled

Table 1. General Characteristics of Patients with $A A^{*}$

\begin{tabular}{|c|c|c|c|}
\hline Characteristic & $\operatorname{AAA}(n=183)$ & TAA $(n=162)$ & $P$ Value \\
\hline Age (y) & 73 (67 to 80 ) & 67 (56 to 78$)$ & .106 \\
\hline Sex & & & .008 \\
\hline Male & $126(68.85)$ & $89(54.93)$ & \\
\hline Female & $57(31.15)$ & $73(45.07)$ & \\
\hline Marital status & & & .005 \\
\hline Single & $18(9.83)$ & $38(23.45)$ & \\
\hline Married & $106(57.92)$ & $90(55.55)$ & \\
\hline Divorced & $14(7.65)$ & $11(6.8)$ & \\
\hline Widowed & $37(20.21)$ & $19(11.72)$ & \\
\hline Other & $8(4.39)$ & $4(2.48)$ & \\
\hline Ethnicity & & & .027 \\
\hline White & $160(87.43)$ & $123(75.92)$ & \\
\hline Black/African American & $5(2.73)$ & $4(2.48)$ & \\
\hline Hispanic/Latino & $3(1.63)$ & $6(3.7)$ & \\
\hline Other & $15(8.21)$ & $29(17.9)$ & \\
\hline Admission type & & & $<.001$ \\
\hline Elective & $132(72.13)$ & $113(69.75)$ & \\
\hline Emergent & $48(26.24)$ & $48(29.62)$ & \\
\hline Urgent & $3(1.63)$ & $1(0.63)$ & \\
\hline In-hospital mortality & $11(6.01)$ & $6(3.7)$ & .231 \\
\hline LOS in ICU (h) & 90.3 (41.9 to 221$)$ & 53.1 (30.2 to 121.8$)$ & $<.001$ \\
\hline APACHEll score & $9(6$ to 11$)$ & $7(5$ to 9$)$ & .035 \\
\hline SOFA score & $1(0$ to 2$)$ & 0.5 (0 to 1$)$ & .045 \\
\hline
\end{tabular}

*Data are mean (SD), median (IQR), or n (\%). 
Table 2. Comparison of Variables between Survivor and Nonsurvivor Groups in AAA*

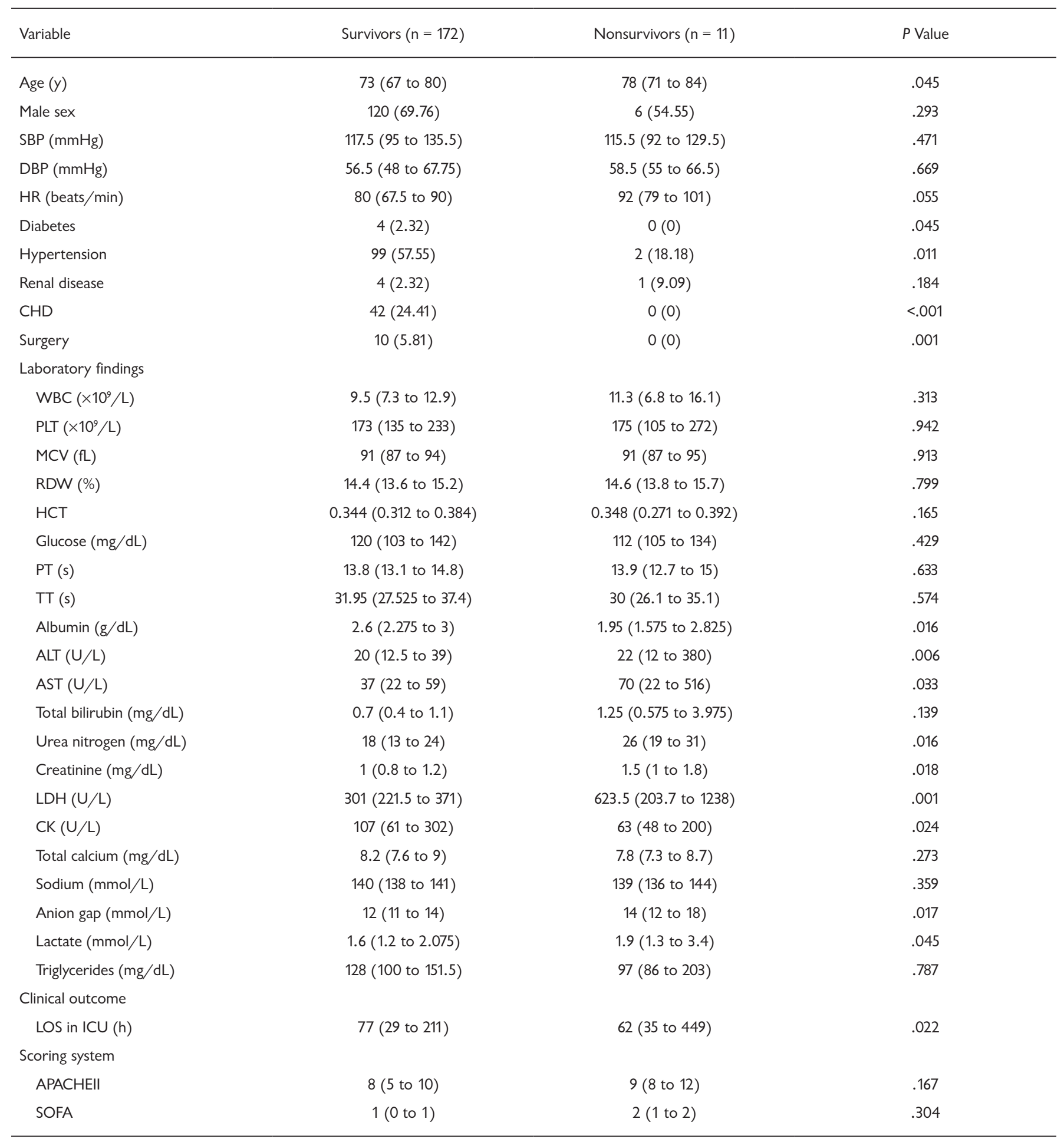

*Data are mean (SD), median (IQR), or n (\%). 
prevalence of AAA [Gratama 2010]. A systemic review on the quality of life in 1272 patients $>75$ years old with AAA reported that perioperative mortality reached $7.8 \%$ [Shan 2019], similar to the results in our study. Moreover, morbidity and mortality increasing significantly with age in AAA was proven in a nationwide German study [Trenner 2020]. In TAA, a decade-long retrospective study in Canada also showed that age was associated with poor clinical outcomes [McClure 2020].

Previous studies have investigated different methods of surgery in AAA and TAA, as well as clinical outcomes $[\mathrm{Li}$ 2019]. Machine learning research with a total of 781 patients with nondissecting ascending TAA showed that patients who did not follow surgical recommendations experienced substantially worse outcomes [Saeyeldin 2019]. A Medicarederived risk prediction model also showed a 10-fold decrease in operative mortality for AAA with surgery compared with nonsurgery [Hicks 2015].

In our study, the level of albumin was significantly lower in the nonsurvivor group, whereas the level of LDH was significantly higher. Hypoalbuminemia has been found to be linked with progression and prognosis in AA [Cersit 2020]. In AAA patients with surgery, preoperative hypoalbuminemia was associated with increased postoperative morbidity and mortality [He 2020]. Moreover, with increasing severity of hypoalbuminemia, early and late mortality in TAA and AAA also increased, as did complications [Inagaki 2017]. Elevated levels of $\mathrm{LDH}$ were associated not only with hypoxia, but also with a marker of inflammation and oxidative stress [Wohlauer 2017]. A clinical prospective study of biochemical markers in patients after thoracic and thoracoabdominal aortic aneurysm surgery showed that elevated $\mathrm{LDH}$ was associated with adverse neurological complications and poor prognosis [Lases 2005].

In MIMIC-III, older AA patients without surgery, with lower levels of albumin, and with higher levels of LDH had higher risk of in-hospital mortality. So far, this is the first study to analyze the clinical characteristics of AA in MIMICIII, providing clinical references for physicians to take early precautions for AA patients with a higher risk of worse clinical outcomes.

\section{Limitations}

Several limitations should be explained. First, MIMIC-III is a public database in the United States. Although the results of this study apply to other countries or ethnicities, caution must be used in doing so. Second, because of relatively small samples, a predictive model could not be constructed. Further research with larger samples in multiple centers should be explored for an accurate model to predict short- and longterm prognosis in AA. Third, some data and variables are missing and incomplete in MIMIC-III, so not all the variables associated with clinical outcomes could be analyzed. Clinical characteristics including etiologies, medical history, operation type, and anatomic location of TAA and AAA were not all included. Previous studies indicated that etiologies including Marfan syndrome, aortic dissection, connective tissue disorders, atherosclerosis, and infection are linked to outcomes
[Li 2021; Boczar 2021]. Other factors such as natural history of different types of aneurysms and laboratory indicators of patients before admission (which were not in the database) also could affect our results. In AAA, the diameter and the location associated with the renal artery definitely affects outcomes. Moreover, TAA in the ascending aorta has a different prognosis from that in the descending aorta [Nargesi 2021; Rueckel 2021]. In the future, prospective research with clinical variables and management needs to be comprehensively analyzed for more clinical guidance.

\section{CONCLUSION}

In this study, age, surgery, albumin, and LDH showed significant differences between survivor and nonsurvivor groups in both AAA and TAA. In the nonsurgery group, the mean age was older and disease severity was worse, with poorer clinical outcomes. Older AA patients without surgery and with lower levels of albumin and higher levels of LDH had higher risk of in-hospital mortality.

\section{REFERENCES}

Acosta S, Gottsater A, Engstrom G, Melander O, Zarrouk M, Nilsson $\mathrm{PM}$, et al. B-type natriuretic peptide for prediction of incident clinically significant abdominal aortic aneurysm: A population-based prospective study. Vasc Med 2018;23:46-51.

Boczar K, Boodhwani M, Beauchesne L, Dennie C, Chan K, Wells $\mathrm{G}$, et al. Aortic stiffness, central blood pressure, and pulsatile arterial load predict future thoracic aortic aneurysm expansion. Hypertension 2021;77:126-134.

Cersit S, Ocal L, Keskin M, Gursoy MO, Kalcik M, Bayam E, et al. Association of C-reactive protein-to-albumin ratio with the presence and progression of abdominal aortic aneurysm. Angiology 2020:3319720954084.

Fattahi N, Rosenblad A, Kragsterman B, Hultgren R. Risk factors in 50 -year-old men predicting development of abdominal aortic aneurysm. J Vasc Surg 2020;72:1337-1346.e1.

Gratama JW, van Leeuwen RB. Abdominal aortic aneurysm: High prevalence in men over 59 years of age with TIA or stroke, a perspective. Abdom Imaging 2010;35:95-98.

Grootes I, Barrett JK, Ulug P, Rohlffs F, Laukontaus SJ, Tulamo R, et al. Predicting risk of rupture and rupture-preventing reinterventions following endovascular abdominal aortic aneurysm repair. Br J Surg 2018;105:1294-1304

He H, Chai X, Zhou Y, Pan X, Yang G. Association of lactate dehydrogenase with in-hospital mortality in patients with acute aortic dissection: A retrospective observational study. Int J Hypertens 2020;2020:1347165.

Hicks CW, Black JH 3rd, Arhuidese I, Asanova L, Qazi U, Perler BA, et al. Mortality variability after endovascular versus open abdominal aortic aneurysm repair in a large tertiary vascular center using a Medicarederived risk prediction model. J Vasc Surg 2015;61:291-297.

Inagaki E, Farber A, Eslami MH, Kalish J, Rybin DV, Doros G, et al. Preoperative hypoalbuminemia is associated with poor clinical outcomes after open and endovascular abdominal aortic aneurysm repair. J Vasc Surg 2017;66:53-63.e1. 
Table 3. Comparison of Variables between Survivor and Nonsurvivor Groups in TAA*

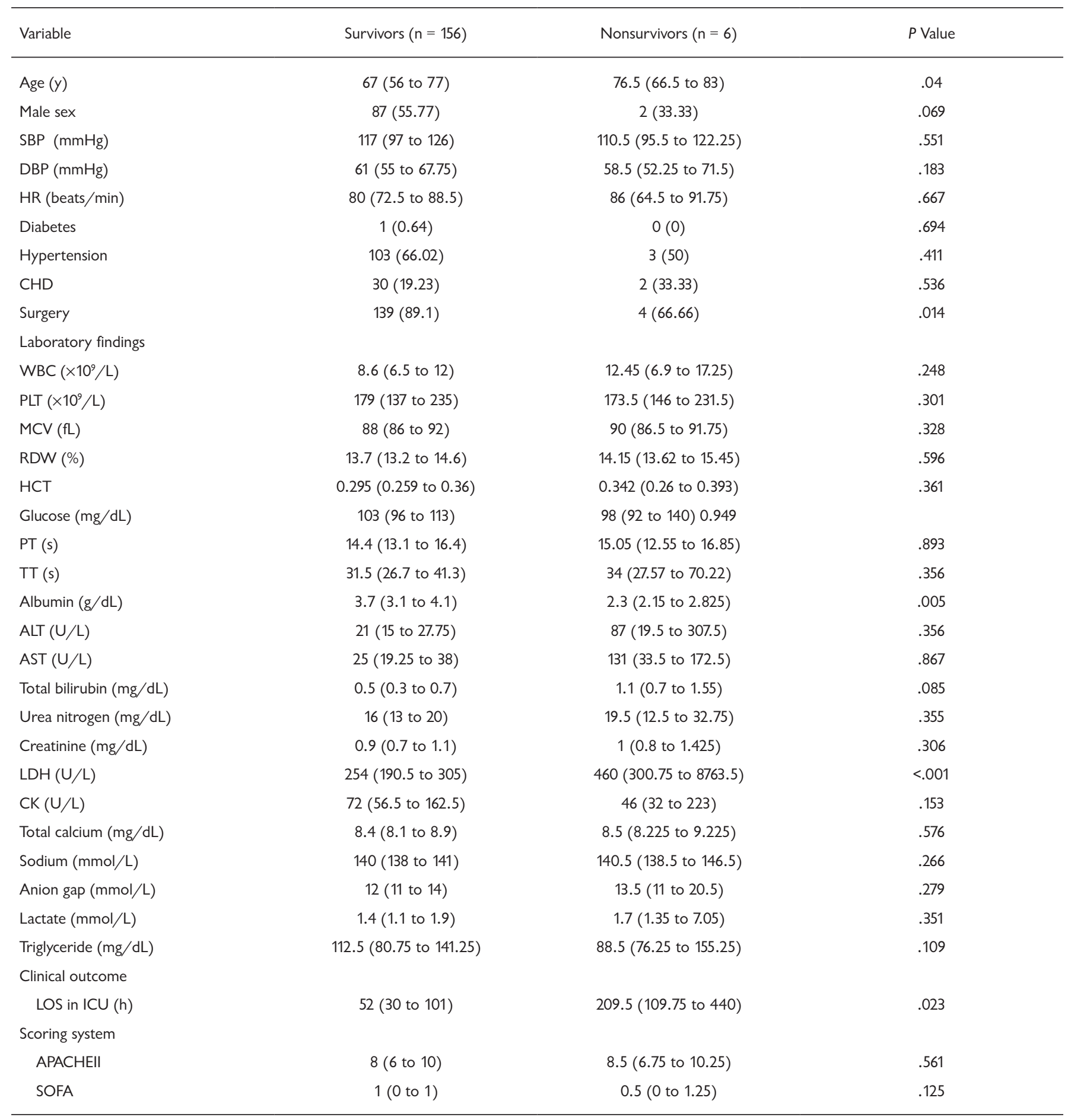

*Data are mean (SD), median (IQR), or n (\%). 
Table 4. Comparison of Variables between Surgery and Nonsurgery Groups*

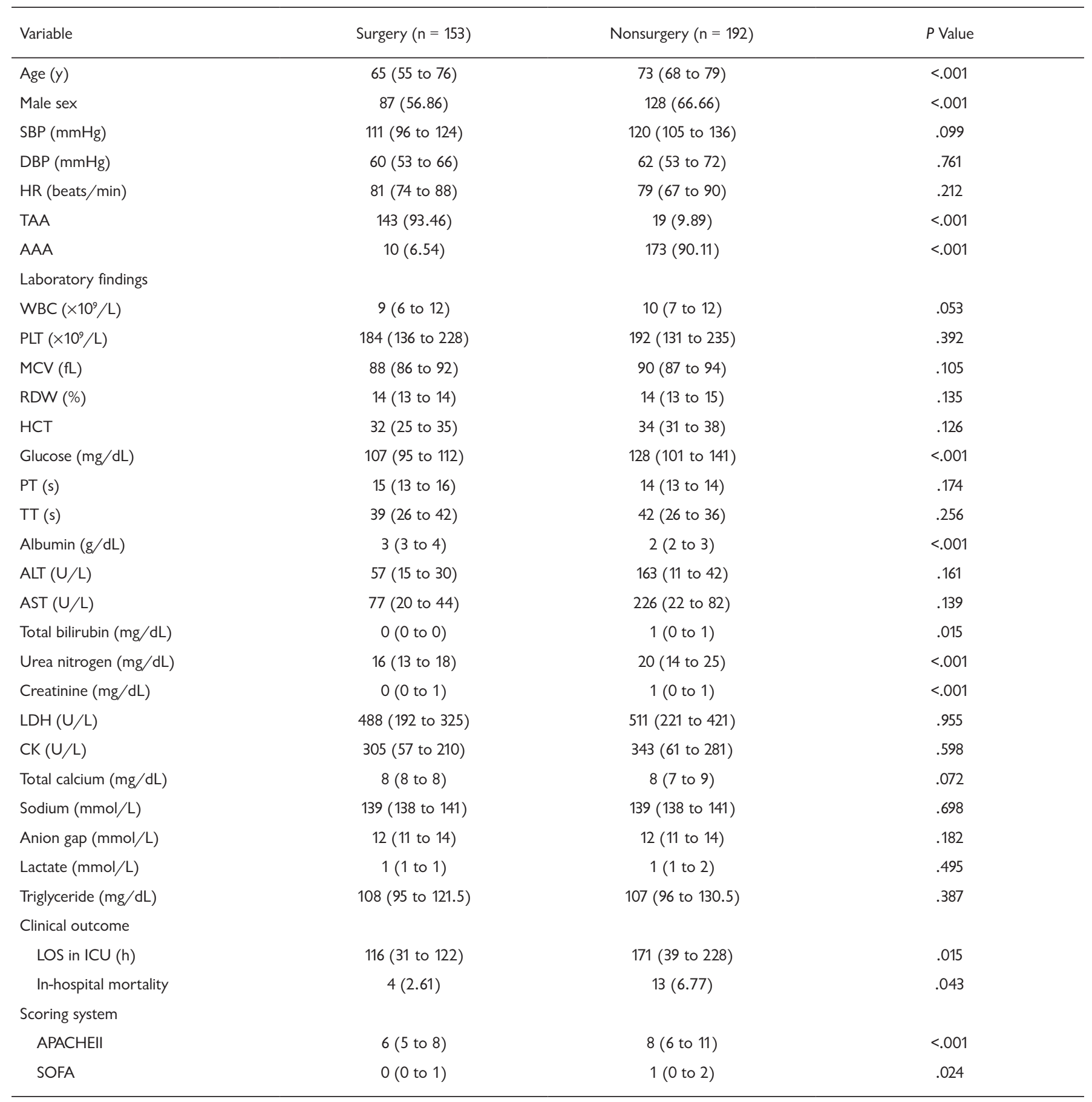

*Data are mean (SD), median (IQR), or n (\%). 
Isselbacher EM. Thoracic and abdominal aortic aneurysms. Circulation 2005;111:816-828.

Johnson AEW, Pollard TJ, Shen L, Lehman LWH, Feng ML, Ghassemi M, et al. MIMIC-III, a freely accessible critical care database. Scientific Data 2016;3.

Jordanski M, Radovic M, Milosevic Z, Filipovic N, Obradovic Z. Machine learning approach for predicting wall shear distribution for abdominal aortic aneurysm and carotid bifurcation models. IEEE J Biomed Health Inform 2018;22:537-544.

Lases EC, Schepens MA, Haas FJ, Aarts LP, ter Beek HT, van Dongen EP, et al. Clinical prospective study of biochemical markers and evoked potentials for identifying adverse neurological outcome after thoracic and thoracoabdominal aortic aneurysm surgery. $\mathrm{Br} \mathrm{J}$ Anaesth 2005;95:651-661.

Leighton P, Doe M, Pathak S, AlDuwaisan A, Brooks M. Immediate impact of centralization on abdominal aortic aneurysm repair outcomes for a vascular network in the south west of England: A retrospective cohort study. Ann Surg 2019;269:172-176.

Li B, Khan S, Salata K, Hussain MA, de Mestral C, Greco E, et al. A systematic review and meta-analysis of the long-term outcomes of endovascular versus open repair of abdominal aortic aneurysm. J Vasc Surg 2019;70:954-969.e30.

Li J, Pan C, Zhang S, Spin JM, Deng A, Leung LLK, et al. Decoding the genomics of abdominal aortic aneurysm. Cell 2018;174:1361-1372.e10.

Li T, Liu X, Sun H, Ning H, Yang J, Ma C. Assessment of the global and regional circumferential strain of abdominal aortic aneurysm with different size by speckle-tracking echocardiography. J Ultrasound Med doi:10.1002/jum.15651.

McClure RS, Brogly SB, Lajkosz K, McClintock C, Payne D, Smith HN, et al. Economic burden and healthcare resource use for thoracic aortic dissections and thoracic aortic aneurysms-A population-based cost-ofillness analysis. J Am Heart Assoc 2020;9:e014981.

Molacek J, Treska V, Zeithaml J, Hollan I, Topolcan O, Pecen L, et al. Blood biomarker panel recommended for personalized prediction, prognosis, and prevention of complications associated with abdominal aortic aneurysm. EPMA J 2019;10:125-135.

Mori M, Yousef S, Zhuo H, Bin Mahmood SU, Mojibian H, Zhang $\mathrm{Y}$, et al. Diabetes and hypertension associate differently with the risk of ascending thoracic aortic aneurysm: A CT study of 21,295 patients. JACC Cardiovasc Imaging 2020;13:1634-1636.
Nargesi S, Abutorabi A, Alipour V, Tajdini M, Salimi J. Cost-effectiveness of endovascular versus open repair of abdominal aortic aneurysm: A systematic review. Cardiovasc Drugs Ther doi:10.1007/ s10557-020-07130-6.

Pasta S, Agnese V, Gallo A, Cosentino F, Di Giuseppe M, Gentile G, et al. Shear stress and aortic strain associations with biomarkers of ascending thoracic aortic aneurysm. Ann Thorac Surg 2020;110:1595-1604.

Pires Coelho A, Lobo M, Brandao JP, Nogueira C, Tournoij E, Jongkind $\mathrm{V}$, et al. Prediction of survival after 48 hours of intensive unit care following repair of ruptured abdominal aortic aneurysm-multicentric study for external validation of a new prediction score for 30-day mortality. Ann Vasc Surg 2019;60:95-102.

Rueckel J, Reidler P, Fink N, Sperl J, Geyer T, Fabritius M, et al. Artificial intelligence assistance improves reporting efficiency of thoracic aortic aneurysm CT follow-up. Eur J Radiol 2021;134:109424.

Saeyeldin A, Zafar MA, Li Y, Tanweer M, Abdelbaky M, Gryaznov A, et al. Decision-making algorithm for ascending aortic aneurysm: Effectiveness in clinical application? J Thorac Cardiovasc Surg 2019;157:1733-1745.

Shan L, Saxena A, Goh D, Robinson D. A systematic review on the quality of life and functional status after abdominal aortic aneurysm repair in elderly patients with an average age older than 75 years. J Vasc Surg 2019;69:1268-1281.

Trenner M, Salvermoser M, Busch A, Reutersberg B, Eckstein HH, Kuehnl A. Effect modification of sex and age for the hospital volume-outcome relationship in abdominal aortic aneurysm treatment: Secondary data analysis of the nationwide German diagnosis related groups statistics from 2005 to 2014. J Am Heart Assoc 2020;9:e014534.

Wang TKM, Desai MY. Thoracic aortic aneurysm: Optimal surveillance and treatment. Cleve Clin J Med 2020;87:557-568.

Wohlauer M, Brier C, Kuramochi Y, Eagleton M. Preoperative Hypoalbuminemia is a risk factor for early and late mortality in patients undergoing endovascular juxtarenal and thoracoabdominal aortic aneurysm repair. Ann Vasc Surg 2017;42:198-204.

Yamada S, Asakura H. Management of disseminated intravascular coagulation associated with aortic aneurysm and vascular malformations. Int J Hematol 2021;113:15-23.

Yigit G, Ozen A, Cetinkaya F, Unal EU, Iscan HZ, Birincioglu CL, et al. Early and late-term follow-up results of patients diagnosed with aortic aneurysm or aortic dissection with aortic regurgitation undergoing aortic valve repair or valve-sparing aortic surgery. Braz J Cardiovasc Surg doi:10.21470/1678-9741-2020-0133. 